\title{
CONTACTLESS ANGLE DETECTION USING PERMALLOY
}

Kees J. Eijkelt and Rolf Rijk

Twente University of Technology

P. 0.Box 217, 7500 AE Enschede, The Netherlands

+ The Netherlands Technology Foundation (STW)

\section{Abstract}

An overview will be given of measurements on angle detectors. The detectors consist of a pair of planarHall elements opposite to a rotatable magnet. The measurements are performed on a number of planar-Hall elements of different shape and size, and show very good agreement with a previously described theoretical model. To increase the accuracy of the angle detector, three modifications of the original concept of the planar-Hall elements are treated:

a: A ring-shaped element having increased output voltage.

b: An optimized configuration of a pair of planar-Hall elements and a permanent magnet, to cancel out the effects of magnetic anisotropy in the permalloy on the accuracy of the angle detector.

c: An annealing step at $500^{\circ} \mathrm{C}$ applied to the permalloy to create a magnetically isotropic film.

The results of measurements on planar-Hall elements as suggested in a, b and $c$ are presented and show good agreement with theory. Magnetization behavior in a ring structure, combining magnetic anisotropy and magnetic form effects, is not yet properly understood.

\section{Introduction}

An angle detector consisting of a pair of planar-Hall elements opposite to a rotatable magnet has been proposed $[1,2]$. In this paper, measurements, carried out to determine the achievable accuracy of the detector, are described. The term "detector error" will be used for the difference between the angle to be measured and the angle derived from the detector output signal. The measurements have been performed on single planar-Hall elements of different shape and size. In a planar-Hall element we have ( $f$ igure 1 ):

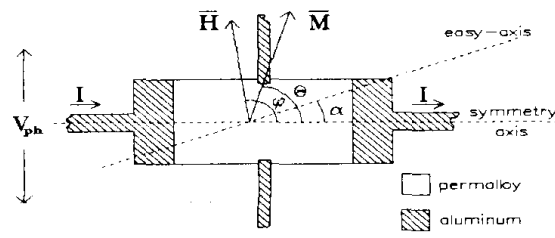

Fig 1: A planar Hall element.

$V_{p h}=\frac{1}{2} I \frac{\Delta \rho}{t} \sin (2 \Theta)$

with I the exciting current, $t$ the film thickness and $\Delta \rho$ the magnetoresistive effect. Other parameters are explained in figure 1 . The current density at a point in the film is considered constant over the film thickness, while the shape of the element may be arbitrary. $\overrightarrow{\mathrm{H}}$ is the external field and $\overline{\mathrm{M}}$ the magnetization in the film. Permalloy thin films show a uniaxial anisotropy. The angle between the easy-axis and the symmetry axis of the device is called $\alpha$. When the permalloy film follows the Stoner-Wohlfarth single domain model [3], the direction of $\overrightarrow{\mathrm{H}}$ and $\overline{\mathrm{M}}$ will follow (2), with $\mathrm{H}_{\mathbf{k}}$ the anisotropy field of the permalloy film.

$\sin (\varphi-\Theta)=\frac{H_{k}}{2 H} \sin 2(\Theta-\alpha)$

The angle detector measures the magnetization angle $\Theta$. Therefore, the uniaxial anisotropy will introduce a systematic detector error $\varphi-\Theta$.

Planar-Hall elements were created on thermally oxidized $\left(0.5 \mu \mathrm{m} \mathrm{SiO}_{2}\right)$ silicon wafers. First an aluminum electrode pattern was realised by deposition of $0.5 \mu \mathrm{m}$ aluminum by evaporation followed by wet chemical etching. The permalloy thin film $(50 \mathrm{~nm})$ was sputtered in an $\mathrm{Ar}$ atmosphere using a bias field of $2000 \mathrm{~A} / \mathrm{m}$ to direct the uniaxial anisotropy. After wet chemical etching of the permalloy, the planar Hall elements were fixed onto epoxy substrates.

The planar-Hall elements were mounted on a rotation stage in a pair of Helmholtz coils which produce a homogeneous external field of up to $6000 \mathrm{~A} / \mathrm{m}$. The rotation stage was used to rotate the element with respect to the field in 180 steps of $1^{\circ}$. The signal voltage $v_{p h}$ was measured for each step and stored by a computer. The computer analysis uses (1) to calculate the magnetization direction as a function of field direction, deriving parameters $A$ (offset of $V_{p h}$ ) and $B$ (amplitude of $\mathrm{v}_{\mathrm{ph}}$ ). The results are compared with the single domain model, fitting the parameters $\alpha$ and $\mathrm{H}_{\mathrm{k}} / \mathrm{H}$. Finally the RMS value $\Delta$ of the deviation between theoretical and measured magnetization direction is calculated.

To characterize the element, the off set to signal voltage ratio $A / B$ is calculated as well as $B / I$, indicating the yield of the element. Details on measurement equipment and analysis are given elsewhere [4].

\section{Modifications}

To increase the accuracy of the angle detector in its basic configuration, three modifications are considered:

a: By changing the form of the planar-Hall element, the output voltage is increased.

b: A system of two planar-Hall elements and a permanent magnet is optimized to cancel out errors in the detector output caused by the magnetic anisotropy of the permalloy.

c: Annealing the permalloy at $500^{\circ} \mathrm{C}$, a magnetically isotropic film is obtained.

\section{Ring-shaped elements}

The output voltage of a planar-Hall element is directly proportional to the current 1 through the element and inversely proportional to the film thickness $t$. Very wide planar-Hall elements should be used to get a significant increase in $V_{p h}$ without exceeding the maximum current density, giving rise to design problems. A Wheatstone bridge as in figure $2 a$, consisting of four magnetoresistors, has an output signal $V_{b}$ which is equivalent to the output of a planar-Hall element:

$\mathrm{V}_{\mathrm{b}}=\frac{1}{2} \mathrm{I} \frac{1 \Delta \rho}{w t} \sin (\gamma) \sin (2 \varphi)$

with $I$ and $w$ the length and width of each magnetoresistor and $\gamma$ as in figure $2 a$. $V_{b}$ has a maximum for $\gamma=90^{\circ}$. In this calculation, no anisotropy effects are considered. $\mathrm{V}_{\mathrm{b}}$ can be increased by changing $1, \mathrm{w}$ and $t$. In an angle detector using these elements, magnetic form effects in the magnetoresistors will introduce a detector error, increasing with decreasing thickness to width ratio $t / w$. A ring shape (figure $2 b$ ) reduces this error considerably. Figure 3 shows the result of a computer simulation for given thickness to width ratio and increasing field. 

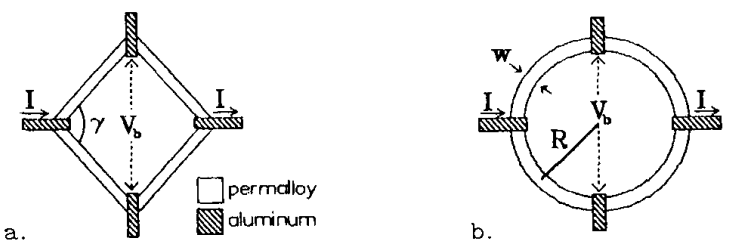

Fig 2a: A Wheatstone bridge of magnetoresistors. Fig 2b: A ring-shaped element.
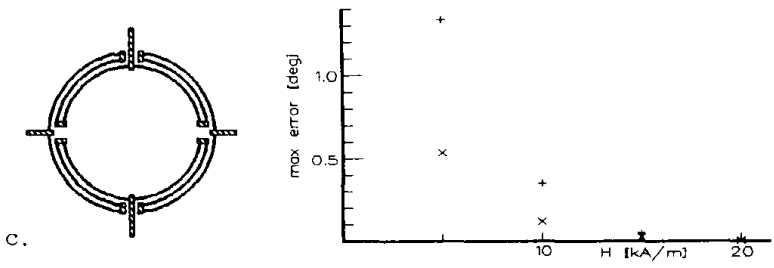

Fig 2c: A three times folded element.

Fig 3 : Maximum detector error for a rectangular bridge $(+)$ and a ring-shaped element $(x), t / w=0.0025$.

One can derive the output voltage for a ring-shaped element:

$\mathrm{V}_{\mathrm{b}}=\frac{1}{2} \mathrm{I} \frac{\Delta \rho}{t} \sin (2 \Theta) \frac{1}{\ln \left(\frac{R+w / 2}{R-w / 2}\right)}$

$R$ is the radius to the half-width of the ring and $w$ is the width of the ring. To further increase the output voltage without consuming too much space, the ring can be folded into a structure as in figure $2 \mathrm{c}$.

\section{Using an inhomogeneous field}

A way to reduce the detector error caused by the magnetic anisotropy of the film material, is to exploit the field inhomogeneity of a permanent magnet [1]. The success of this method depends on the value of several adjustable parameters.

The field strength of the magnet determines the influence of the anisotropy. On the other hand, the influence of the field inhomogeneity depends on the shape of the magnet, the relation between the

dimensions of the magnet and the sensor (= planar-Hall pair), and the distance between them.

A model of a magnet-sensor system has been made. The magnetic charge is assumed to be homogeneously distributed on two opposite faces of the magnet. The StonerWohlfarth single domain model is used and the current distribution in the circular films is approximated with an ellipsoidal pattern. In a computer simulation, this model was used to optimize all parameters.

A configuration, consisting of a permanent magnet $\left(50 \times 50 \times 8 \mathrm{~mm}^{3}\right.$, magnetization $\left.480 \mathrm{kA} / \mathrm{m}\right)$ and a sensor comprising two circular planar-Hall elements (ø $6.7 \mathrm{~mm}$, distance between centres $8 \mathrm{~mm}$ ) at $16 \mathrm{~mm}$ beneath the magnet surface, was found appropriate.

\section{Isotropic permalloy films}

Another way to suppress the effects of magnetic anisotropy on the detector output is to modify the permalloy itself. Isotropic permalloy films can be produced by disarranging the easy-axis orientation in different parts of the film. When the film consists of a great number of small areas $A^{*}$, each with its own randomly oriented uniaxial anisotropy and anisotropy field strength $H_{k}^{x}\left(0<H_{k}^{x}<H_{k}^{\max }\right)$, the film as a whole will not have an easy-axis. When a homogeneous external field $\overline{\mathrm{H}}$ is switched on $\left(\mathrm{H}>\mathrm{H}_{\mathrm{k}}^{\max }\right)$, the distribution of magnetization directions in the areas $A^{x}$ will be symmetrical with respect to the field direction.
Therefore, the total magnetization $M$ in the film will have the same direction as $\overline{\mathrm{H}}$ but will be smaller than $M_{s}$, the saturation magnetization. The anisotropyinduced detector error will be completely canceled out in this way. We tried an annealing treatment at $500^{\circ} \mathrm{C}$ and zero field to disarrange the easy-axis orientation.

\section{Measurements and discussion}

The result of a typical measurement on a planar-Hall element is depicted in figure 4 . The element has a circular shape $(\curvearrowleft 10 \mathrm{~mm})$ and four contacts around the circumference, overlapping $100 \times 100 \mu^{2}$ with the film. The difference $\varphi-\Theta$ between field direction and magnetization direction in the film, caused by the magnetic anisotropy, is plotted against the position of the rotation stage over the complete range of the angle detector. The crosses represent the measured values and are derived using (1). For clarity of the plot, not all 180 measurement points are shown. With (2), representing the single domain model, $\alpha$ and $\mathrm{H}_{\mathrm{k}}$ are calculated from these results. A theoretical curve for $\varphi-\Theta$ is determined, represented by the solid line in figure 4 The RMS value $\Delta$ of the deviation between the measured and theoretical values of $\varphi-\Theta$ is finally derived.

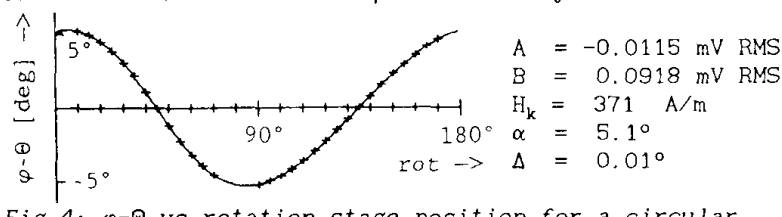

Fig 4: $\varphi-\Theta$ Vs rotation stage position for a circular element $(\varnothing 10 \mathrm{~mm}), H=2000 \mathrm{~A} / \mathrm{m}, I=1.5 \mathrm{~mA}$ RMS

\begin{tabular}{|c|c|c|c|c|c|c|c|}
\hline no & $\begin{array}{c}\mathrm{H} \\
{[\mathrm{A} / \mathrm{m}]}\end{array}$ & $\begin{array}{l}\mathrm{B} / \mathrm{I} \\
{[\Omega]}\end{array}$ & $\mathrm{A} / \mathrm{B}$ & $\begin{array}{c}\mathrm{H}_{\mathrm{k}} \\
{[\mathrm{A} / \mathrm{m}]}\end{array}$ & $\begin{array}{c}\alpha \\
{[0]}\end{array}$ & $\begin{array}{c}\Delta \\
{\left[\begin{array}{c}0 \\
\end{array}\right]}\end{array}$ & $\begin{array}{l}\text { permalloy } \\
\text { geometry }\end{array}$ \\
\hline 1 & 2000 & 0.0612 & -0.125 & 371 & 5. 1 & 0.01 & $\varnothing 10 \mathrm{~mm}$ \\
\hline 2 & 2000 & 0.0936 & -0.818 & 474 & 130.2 & 0.02 & $\varnothing \quad 1$ \\
\hline 3 & 2000 & 0.0526 & 0.025 & 409 & 96.0 & 0.01 & $\mathrm{~mm}$ \\
\hline 4 & 2000 & 0.0604 & 0.083 & 386 & 82.4 & 0.09 & \\
\hline
\end{tabular}

Table 1: Measurement results for planar-Hall elements, showing field strength $H$, yield $B / I$, offset ratio $A / B$, anisotropy parameters $H_{\mathbf{k}}$ and $\alpha$, RMS value of the deviation from the model $\Delta$ and the geometry of the element.

In table 1, the results are given for four different geometries. It should be noted, that smaller size does not affect the magnetic behavior of the element. The fourth measurement shows a significant deviation from the model, caused by magnetic form effects. The permalloy in this element has circular shape (ø $1 \mathrm{~mm}$ ). with extensions of $250 \mathrm{~m}$ length leading to the contacts. These extensions are $100 \mu \mathrm{m}$ and $250 \mu \mathrm{m}$ wide for the voltage and current contacts respectively.

Measurements on ring-shaped elements are shown in figure $5 a$ and table 2 for different radius to ringwidth ratio $R / w$.
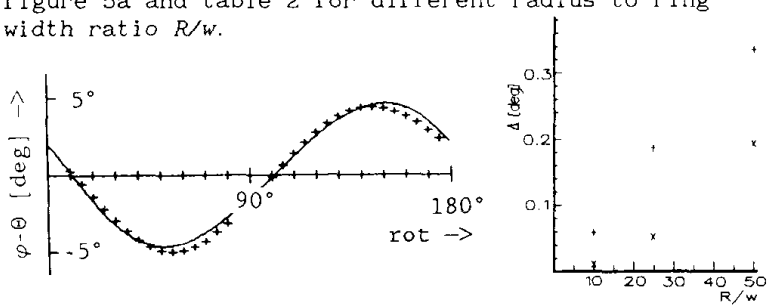

Fig 5a: $\varphi-\theta$ vs rotation stage position for a ring with $R / w=50, H=2000 \mathrm{~A} / \mathrm{m}$.

Fig 5b: Measured ( + ) and theoretical ( $x$ ) values for $\Delta$ for different $R / W$ at $H=2000 \mathrm{~A} / \mathrm{m}$ 


\begin{tabular}{|c|c|c|c|c|c|c|}
\hline no & $\begin{array}{c}\mathrm{H} \\
{[\mathrm{A} / \mathrm{m}]}\end{array}$ & $\begin{array}{c}\mathrm{B} / \mathrm{I}[\Omega] \\
\quad \exp \\
\end{array}$ & \begin{tabular}{|l|l}
$/ I$ & {$[\Omega]$} \\
theory
\end{tabular} & $\mathrm{A} / \mathrm{B}$ & $\begin{array}{c}\Delta \\
{\left[{ }^{\circ}\right]}\end{array}$ & $\bar{R} / \mathrm{W}$ \\
\hline 1 & 2000 & 0.451 & 0.428 & 0.681 & 0.06 & 10 \\
\hline 2 & 2000 & 1.107 & 1.106 & 1.138 & 0.19 & 25 \\
\hline 3 & 2000 & 11.17 & 11.17 & -0.247 & 0.32 & *50 \\
\hline
\end{tabular}

Table 2: Measurement results for ring-shaped elements. Anisotropy parameters have been left out, because the single domain model is not valid. The theoretical yield $B / I$ of the elements is calculated from (4), with the factor $\Delta \rho / t$ derived from the third measurement. *: This element is a five times folded type.

The periodic deviation from the model in $5 a$ is caused by the ring shape, but is larger than expected. In the previous discussion, only magnetic form effects were considered. Therefore the combination of intrinsic and form anisotropy [5] in a ring-shaped element was simulated. The results are depicted in figure $5 \mathrm{~b}$, together with the measured results. It is clear that the extended model is not sufficient. In the model, the amplitude of the deviations is directly proportional to the square of the ratio of $R / w$ to $H$. In the measurements a linear dependence is found.

To verify the model for inhomogeneous fields, a magnetsensor system as described in the introduction was realised. The magnet was mounted on a rotation stage, rotating over 360 steps of $1^{\circ}$. The position of the magnet and the sensor with respect to the rotation axis of the stage could be changed. Also the distance between the magnet and the sensor could be adjusted. For all 360 positions of the magnet, the signal voltage of a single planar-Hall element was measured. In a complete angle detector, the output signal consists of a pair of planar-Hall voltages. With the measured voltage and a planar-Hall voltage calculated for an ideal element $(\varphi \equiv \Theta)$, a complete angle detector was simulated, and the detector error was determined for the total measurement range (see figure 6). The detector error was minimized by adapting the adjustable geometric parameters in the magnet-sensor system.

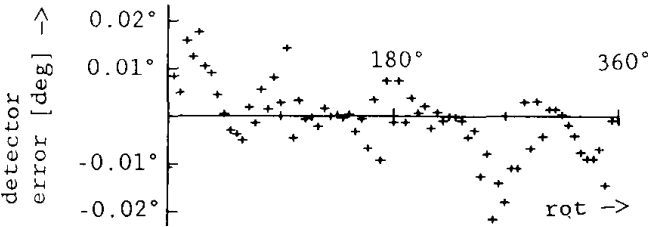

Fig 6: Detector error of a magnet-sensor system.

For a real angle detector the detector error can be calculated by simply adding the results of similar measurements, performed separately on the two planarHall elements of the detector.

The distance between the surface of the magnet and the sensor is $14 \mathrm{~mm}$. The field of the magnet was found to be smaller than expected, explaining this lower optimum distance. The field strength in the sensor plane is 18 $\mathrm{kA} / \mathrm{m}$. The maximum detector error is less than $0.03^{\circ}$. It should be noted that after minimizing the detector error for one planar-Hall element, the other element may not be in its optimum position. In our case, a measurement on the other element at the same sensor position showed an error with a maximum of $0.3^{\circ}$. The error could be 1 imited to $0.1^{\circ}$ for both elements. Applying this method to increase the accuracy of an angle detector, one has to take into account that symmetry of the field of the magnet as well as accurate positioning of magnet and detector with respect to the rotation axis is of high importance. The sensitivity of the detector error for deviations in these positions is about $0.3^{\circ} / \mathrm{mm}$ in our magnet-sensor system.
Figure 7 shows the results of measurements on two planar-Hall elements with permalloy that had been annealed at $500^{\circ} \mathrm{C}$. Anisotropy parameters can not be derived because the single domain model is not valid.

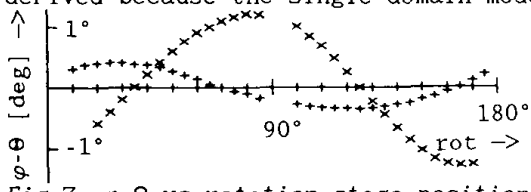

Fig 7: $\varphi-\Theta$ vs rotation stage position for permalloy annealed at $500^{\circ} \mathrm{C}$ for 0.5 hour $(x)$ and 1.5 hour (+). $H=2000 \mathrm{~A} / \mathrm{m}$.

It is clear that the maximum error in the (average) magnetization direction decreases with increasing annealing time. A measurement, using the Crowther method [6], showed a very high angular dispersion in these films. $\alpha_{90}$ was estimated to be more than $80^{\circ}$ and $100^{\circ}$ for the films annealed for 0.5 hour and 1.5 hour respectively. Measurements at $\mathrm{H}=6000 \mathrm{~A} / \mathrm{m}$ on a planar-Hall element with permalloy annealed for 1.5 hour at $500^{\circ} \mathrm{C}$ shows a maximum value for $\varphi$ - $\Theta$ of $0.1^{\circ}$. An angle detector consisting of a pair of these elements and using a magnetic field of $10 \mathrm{kA} / \mathrm{m}$ or more will show a total error of well below $0.1^{\circ}$ for the total range. when of fset voltages are not considered.

\section{Conclusions}

Measurements have been performed on a number of planarHall elements. A theoretical model, combining the Stoner-Wohlfarth single domain model and the planarHall effect in the structure, accurately describes the measurement results.

Ring-shaped elements show an increased output voltage. Magnetization behavior in a ring structure, combining magnetic anisotropy and magnetic form effects, is not yet properly understood.

Errors in the output of an angle detector, caused by magnetic anisotropy, may be reduced considerably by exploiting the inhomogeneity of the field of a permanent magnet.

Annealing at $500^{\circ} \mathrm{C}$ increases angular dispersion in easy-axis orientation in a permalloy film, reducing the errors caused by magnetic anisotropy in an angle detector.

\section{Acknowledgments}

This work is supported by the Netherlands Technology Foundation (STW). The authors would like to thank the Centre for Micro Electronics Twente for their support. We also thank prof.dr.J.H.J. Fluitman, dr.ir.W.H.G Horsthuis, ir. H. Leeuwis and ir.R.M. de Ridder for helpful discussions.

\section{References}

[1] K.J. Eijkel and J.H. Fluitman, Angle detection based on the resistance anisotropy of permalloy, IEEE Trans. Magn. MAG-22, pp. 955-957 (1986).

[2] C.J.M. Eijkel aind J.H. J. Fluitman, Contactless angle transducer.

US Patent Application 06/913,760, september 1986.

[3] M. Prutton, Thin Ferromagnetic Films. London: Butterworth, 1964, p. 55.

[4] K.J. Eijkel, Measurement of the anisotropy in permal loy, IEEE Trans. Magn., this issue, (march 1988)

[5] J.H.J. Fluitman, The influence of sample geometry on the magnetoresistance of $\mathrm{Ni}-\mathrm{Fe}$ films, Thin Solid Films 16, pp. 269-276 (1973).

[6] T.S. Crowther, Angular and magnitude dispersion of the anisotropy in magnetic $\mathrm{films}$, Journal of Applied Physics 34, pp. $580-587$ (1963). 